\title{
Recent genome reduction of Wolbachia in Drosophila recens targets phage WO and narrows candidates for reproductive parasitism
}

Wolbachia are maternally transmitted endosymbionts that often alter their arthropod hosts' biology to favor the success of infected females, and may also serve as a speciation microbe driving reproductive isolation. Two of these host manipulations include killing males outright or causing markedly reduced offspring survival when infected males mate with uninfected females, a phenomenon known as cytoplasmic incompatibility. Little is known about the mechanisms behind these phenotypes, but interestingly either effect can be caused by the same Wolbachia strain when infecting different hosts. For instance, $w$ Rec causes cytoplasmic incompatibility in its native host Drosophila recens and male killing in $D$. subquinaria. The discovery of prophage WO elements in most arthropod Wolbachia has generated the hypothesis that WO may encode genes involved in these reproductive manipulations. However, PCR screens for the WO minor capsid gene indicated that $w$ Rec lacks phage WO. Thus, wRec seemed to provide an example where phage WO is not needed for Wolbachiainduced reproductive manipulation. To enable investigation of the mechanism of phenotype switching in different host backgrounds, and to examine the unexpected absence of phage WO, we sequenced the genome of $w$ Rec. Analyses reveal that $w$ Rec diverged from $w \mathrm{Mel}$ approximately 350,000 years ago, mainly by genome reduction in the phage regions. While it lost the minor capsid gene used in standard PCR screens for phage WO, it retained two phage WO regions encompassing 33 genes, several of which have previously been associated with reproductive parasitism. Thus, WO gene involvement in reproductive manipulation cannot be excluded and reliance on single gene PCR should not be used to rule out the presence of phage WO in Wolbachia. Additionally, the genome sequence for $w$ Rec 
will enable transcriptomic and proteomic studies that may help elucidate the Wolbachia mechanisms of altered reproductive manipulations associated with host switching, perhaps among the 33 remaining phage genes. 


\section{Recent Genome Reduction of Wolbachia in Drosophila recens Targets Phage 2 WO and Narrows Candidates for Reproductive Parasitism}

3 Jason A. Metcalf ${ }^{1}$, Minhee Jo ${ }^{1}$, Sarah R. Bordenstein ${ }^{1}$, John Jaenike ${ }^{2}$, Seth R. Bordenstein ${ }^{1,3, *}$

$4 \quad{ }^{1}$ Department of Biological Sciences, Vanderbilt University, Nashville, TN 37232, USA.

$5 \quad{ }^{2}$ Depatment of Biology, University of Rochester, Rochester, NY 14627, USA

$6 \quad{ }^{3}$ Department of Pathology, Microbiology, and Immunology, Vanderbilt University, Nashville, TN 7 37232, USA.

8 *Correspondence and requests for materials should be addressed to:

9 s.bordenstein@vanderbilt.edu

10 Abstract: 
11 Wolbachia are maternally transmitted endosymbionts that often alter their arthropod hosts'

12 biology to favor the success of infected females, and may also serve as a speciation microbe

13 driving reproductive isolation. Two of these host manipulations include killing males outright or

14 causing markedly reduced offspring survival when infected males mate with uninfected females,

15 a phenomenon known as cytoplasmic incompatibility. Little is known about the mechanisms

16 behind these phenotypes, but interestingly either effect can be caused by the same Wolbachia

17 strain when infecting different hosts. For instance, $w$ Rec causes cytoplasmic incompatibility in its

18 native host Drosophila recens and male killing in D. subquinaria. The discovery of prophage WO

19 elements in most arthropod Wolbachia has generated the hypothesis that WO may encode genes

20 involved in these reproductive manipulations. However, PCR screens for the WO minor capsid

21 gene indicated that $w$ Rec lacks phage WO. Thus, $w$ Rec seemed to provide an example where

22 phage WO is not needed for Wolbachia-induced reproductive manipulation. To enable

23 investigation of the mechanism of phenotype switching in different host backgrounds, and to

24 examine the unexpected absence of phage WO, we sequenced the genome of $w$ Rec. Analyses

25 reveal that $w$ Rec diverged from $w$ Mel approximately 350,000 years ago, mainly by genome

26 reduction in the phage regions. While it lost the minor capsid gene used in standard PCR screens

27 for phage WO, it retained two phage WO regions encompassing 33 genes, several of which have

28 previously been associated with reproductive parasitism. Thus, WO gene involvement in

29 reproductive manipulation cannot be excluded and reliance on single gene PCR should not be

30 used to rule out the presence of phage WO in Wolbachia. Additionally, the genome sequence for

$31 w$ Rec will enable transcriptomic and proteomic studies that may help elucidate the Wolbachia

32 mechanisms of altered reproductive manipulations associated with host switching, perhaps

33 among the 33 remaining phage genes.

\section{Introduction:}

PeerJ reviewing PDF | (v2014:07:2386:1:1:NEW 29 Jul 2014) 
Wolbachia are widespread obligate3intracellular $\alpha$-proteobacteria that infect around $40 \%$ of arthropod species ( $\underline{\text { ug and Hammerstein, 2012) }}$ ) and 47\% of filarial nematodes (Ferri et

37 al., 2011). These infection frequencies, if extrapolated to the diversity and abundance of their 38 hosts, make Wolbachia perhaps the most widespread endosymbiont in animals. To maximize its 39 propagation in arthropods, the maternally inherited Wolbachia has evolved an assortment of mechanisms to distort its host's reproductive system in a manner that enhances the relative

41 production of infected females. These mechanisms include feminization, parthenogenesis, male

42 killing, and cytoplasmic incompatibility (CI), the most common phenotype and one that results in

43 embryonic lethality when matings occur between infected males and uninfected females (Werren

44 et al., 2008). Females harboring the same Wolbachia strain, meanwhile, can successfully mate

45 and produce infected offspring with either infected or uninfected males, giving these females a

46 selective advantage in populations of mixed infection status.

47 Interestingly, some Wolbachia strains are multipotent and can induce more than one type of 48 reproductive manipulation depending on the arthropod host it infects (Fujii et al., 2001, Jaenike. 49 2007). In one striking example, the Wolbachia strain $w$ Rec causes CI in its native host,

50 Drosophila recens, but when introgressed into a sibling species, D. subquinaria, it causes male

51 killing (Jaenike, 2007). Moreover in a natural hybrid zone between these same two species, 52 unidirectional CI plays a major role in reducing interbreeding and thus contributes to

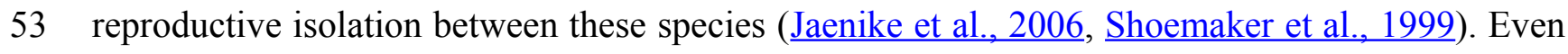

54 though the link between Wolbachia and CI has been known for over 40 years (Yen and Barr. 55 1971), the mechanisms by which Wolbachia accomplishes its reproductive manipulations remain 56 unknown.

57 Despite the physical isolation resulting from its intracellular lifestyle, Wolbachia in

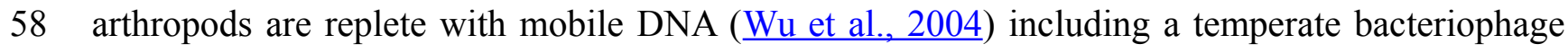
59 named WO (Kent and Bordenstein, 2010, Metcalf and Bordenstein, 2012, Masui et al., 2000). It 
60 has been speculated that WO may be involved in Wolbachia reproductive manipulations due to

61 the prevalence of ankyrin repeat genes in its genome ( $\mathrm{Wu}$ et al., 2004), the pervasiveness of

62 phage-encoded bacterial virulence factors (Boyd, 2012), and the frequent occurrence of phage

63 WO in arthropod Wolbachia strains (Gavotte et al., 2007). However, evidence not supportive of

64 this hypothesis includes the observations that CI is inconsistently associated with the presence of

65 phage WO genes ( $\underline{\text { Sanogo et al., 2005 }} \underline{\text { Saridaki et al., 2011) }}$ ) and that the penetrance of CI in

66 Nasonia wasps is negatively correlated with densities of phage WO virions, as predicted by the

67 phage density model (Bordenstein et al., 2006, Bordenstein and Bordenstein, 2011). Interestingly,

68 PCR screening for the WO minor capsid gene specified WO's absence in $w$ Rec (Bordenstein and

69 Wernegreen, 2004), even though its closest relatives have large amounts of prophage DNA (Wu

70 et al., 2004, Klasson et al., 2009). Thus, the absence of phage WO in $w$ Rec would be a critical

71 example of a Wolbachia strain causing multiple reproductive phenotypes but lacking WO.

72 To investigate the apparent lack of prophage WO genes and alternative genetic mechanisms

73 behind $w$ Rec's diverse phenotypic influences, we sequenced the $w$ Rec genome using next-

74 generation sequencing technology with partial finishing via Sanger sequencing. We determined

75 that although $w$ Rec lacks the WO minor capsid gene typically used in diagnostic screens, it does

76 contain a number of prophage WO genes. Thus, the possibility that WO influences Wolbachia

77 reproductive manipulations cannot be eliminated, and those WO genes present in $w$ Rec offer a

78 streamlined candidate list of the WO genes that could cause reproductive parasitism.

79 Additionally, the availability of genomic information for a Wolbachia strain that is known to

80 switch reproductive phenotypes will enable genomic, transcriptomic, and proteomic approaches

81 to investigate the mechanisms behind these phenotypes.

\section{Materials \& Methods:}


83 The $w$ Rec genome sequencing reads and annotated contigs can be accessed from NCBI

84 Bioproject PRJNA254527.

\section{Wolbachia strain relatedness}

86 Multi-locus sequence typing (MLST) genes were concatenated and a Bayesian phylogeny

87 was inferred as previously described (․Bldo et al., 2006). Briefly, selected fragments of MLST

88 genes (coxA, gatB, fbpA, ftsZ, and hcpA) from Wolbachia strains with complete or nearly

89 complete genome sequences were obtained from GenBank or the sequencing group's online

90 repositories, concatenated for a total length of 2,079bp, and aligned with MUSCLE (Edgar,

91 2004). jModelTest 2 (Darriba et al., 2012) was used to determine the best model of evolution for

92 the set of MLST haplotypes (GTR + I + G), and a Bayesian phylogeny was inferred using Mr.

93 Bayes (Ronquist et al., 2012) with a chain length of $1,100,000$, burn-in of 100,000 , and

94 subsampling frequency of 200.

\section{Genome sequencing and assembly}

96 DNA was extracted from a pool of 10 female Wolbachia infected Pittsford strain $D$.

97 recens flies using a Puregene DNA purification kit (Qiagen, Venlo, Limburg). Shotgun

98 sequencing of the $w$ Rec genome was conducted using an Illumina Hi-Seq (Vanderbilt Sequencing

99 Core, Nashville, TN) with 100 bp paired end sequencing. Reads were filtered using five available

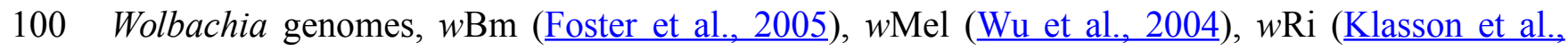

101 2009), wOo (Darby et al., 2012), and wPip (Klasson et al., 2008), by mapping reads to these

102 genomes with length and similarity fractions of 0.5 and keeping all mapped reads, using CLC

103 Genomics Workbench version 6.0.4 (CLC Inc, Aarhus, Denmark). A de novo assembly with a

104 length fraction of 0.5 and similarity fraction of 0.8 was then performed on filtered reads.

105 Sequencing of whole $w$ Rec-infected $D$. recens females produced over 24 million reads, of which 
106

107 Wolbachia-filtered reads yielded 159 contigs. A de novo assembly of unfiltered reads was also

108 performed and any contigs with a portion of its sequence matching contigs obtained from the

109 filtered assembly were added to scaffolds in a search for novel genes. Separately, reads were

110 mapped to the $w \mathrm{Mel}$ genome with length and similarity fractions of 0.5 producing a rough

111 consensus sequence to guide assembly of the de novo contigs into scaffolds, which were further

112 refined with Sanger sequencing of PCR amplifications using primers designed to bind either end 113 of putatively adjacent contigs to yield a final draft genome consisting of 43 scaffolds.

\section{Annotation and comparative genomics}

The $w$ Rec genome was annotated using MicroScope (Vallenet et al., 2009), supplemented with manual curation based on homology with wMel. A comparison of gene-gene identity between $w \mathrm{Mel}$ and $w \mathrm{Rec}$ was performed with a reciprocal best BLAST as previously described (Moreno-Hagelsieb and Latimer, 2008). For whole-genome alignments and analyses, wRec scaffolds were concatenated in the order in which the majority of their genes appear in $w$ Mel. Whole-genome alignment was performed with Mauve (Darling et al., 2010) and a circular

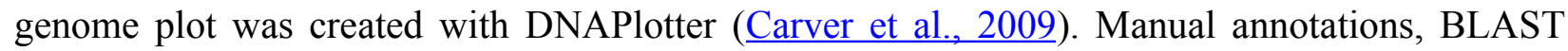
searches, and sequence manipulation were performed with either CLC Genomics Workbench or Geneious V5.5.6 (Biomatters Ltd., Auckland, New Zealand). Ka/Ks rates and ratios were calculated using either single gene or concatenated whole genome CDS alignments with any alignments shorter than 30 amino acids removed (Buschiazzo et al., 2012), using the program DnaSP (Librado and Rozas, 2009). Genomic synteny was assessed with the Cloud Virtual Resource (CloVR) comparative pipeline (Angiuoli et al., 2011) and Sybil synteny gradient viewer (Riley et al., 2012) using the Data Intensive Academic Grid (DIAG) at the University of Maryland. The number of phage and phage-associated genes in Wolbachia genomes was 
130 determined based on current GenBank annotations and includes genes in the phage-packaged

131 eukaryotic association module (Sarah Bordenstein, unpublished data).

\section{Results:}

133 Taxonomy of $w R e c$

134 Phylogenetic analysis based on the concatenated multilocus sequence typing (MLST)

135 genes (Baldo et al., 2006) confirms several previous reports that the supergroup A strain $w$ Rec is 136 closely related to $w \operatorname{Mel}$ (Baldo et al., 2006, Werren et al., 1995, Ioannidis et al., 2007, Gueguen et 137 al., 2012), a widespread strain infecting D. melanogaster (Fig. 1). In addition, phylogenetic 138 analyses of each individual MLST gene support the same relationship of $w$ Mel as the closest 139 sequenced relative to $w \operatorname{Rec}$ (data not shown). To date, all sequenced Wolbachia genomes in 140 supergroups $\mathrm{A}$ and $\mathrm{B}$, including $w \mathrm{Mel}(\underline{\mathrm{Wu}}$ et al., 2004), have contained significant amounts of 141 phage WO DNA. Thus the potential absence of WO in $w$ Rec was unexpected and precipitated the 142 genomic analysis described below.

\section{Genome features of wRec with targeted reduction of prophage WO}

$144 \quad$ Full sequencing statistics and an overview of $w$ Rec genome features are listed in Table 1.

$145 w$ Rec scaffolds $(\mathrm{N}=43)$ consisted of a total sequence length of $1,126,653$ bp containing 1271

146 protein coding sequences. $99.7 \%$ of all nucleotides in coding sequences shared between $w$ Rec 147 and $w \mathrm{Mel}$ were identical, indicating little divergence between these two closely related genomes

148 despite occupying hosts that diverged $>50$ million years ago ( 149 previously established rate of synonymous substitution in Wolbachia of $0.9 \%$ per million years 150 (Raychoudhury et al., 2009), the genome-wide percentage of synonymous substitution $(0.314 \%)$

151 between $w \operatorname{Rec}$ and $w \mathrm{Mel}$ puts their divergence at approximately 350,000 years ago. There were 152 2,009 single nucleotide polymorphisms (SNPs) between shared coding genes in $w \mathrm{Mel}$ and $w$ Rec, 
153 and $599(29.8 \%)$ of these SNPs were synonymous with an average $\mathrm{K}_{\mathrm{a}} / \mathrm{K}_{\mathrm{s}}$ ratio for each gene of

1540.691 . The vast majority of genes are highly conserved between $w \operatorname{Rec}$ and $w$ Mel. More than $95 \%$

155 of orthologous gene pairs were $99 \%$ identical or greater and only ten gene pairs were less than

$15698 \%$ identical (Table 2). Most of these divergent genes code for hypothetical proteins and ankyrin

157 repeat domain proteins. The wsp surface antigen, a known hypervariable sequence in Wolbachia

158 (Zhou et al., 1998), was also among the less conserved loci. All ten divergent genes contained

159 insertions or deletions compared to $w \mathrm{Mel}$ in addition to one or more SNPs. Interestingly, four of

160 these divergent genes, two coding for hypothetical proteins, an Ovarian Tumor (OTU)-like

161 cysteine protease, and $w s p$, had $\mathrm{K}_{\mathrm{a}} / \mathrm{K}_{\mathrm{s}}$ ratios greater than one (Table 2), suggesting that they are

162 evolving under positive selection, and the proteins they encode may be relevant to strain-specific

163 host interactions. When these four genes were aligned to their homologs in $w$ VitA, the closest

164 relative of $w \mathrm{Mel}$ and $w \mathrm{Rec}$, a roughly equal number of mutations in the OTU protease and $w s p$

165 genes in each strain matched the sequence in $w$ VitA. However, for the two hypothetical proteins

166 WREC_0649 (WD_0722) and WREC_1268 (WD_1278), the $w$ Mel alleles matched $w$ VitA in a

167 majority of cases (18 out of 25 nucleotides and 49 out of 56 nucleotides, respectively), suggesting

168 that the $w \mathrm{Mel}$ variants were ancestral and that these $w$ Rec alleles experienced lineage-specific

169 positive selection during $D$. recens infection.

170 Interestingly, there were only two $w$ Rec genes without nucleotide homology to genes in

$171 w \mathrm{Mel}$, even when contigs from a de novo assembly of raw host/Wolbachia reads were mapped to

172 scaffolds in a search for additional genes. These two genes, WREC_0318 and WREC_0319, are

173 hypothetical proteins with $>95 \%$ nucleotide identity to sequences in two other Wolbachia strains,

$174 w \mathrm{Ri}$ and $w \mathrm{Ha}$. Meanwhile, $w$ Rec lacked any homologs of $43 w$ Mel genes (Table S1), all but one

175 of which are phage-related (phage genes discussed below). The single non-phage gene without

176 homology in $w$ Rec is WD_0032, which codes for a hypothetical protein with 96\% similarity to

177 the C-terminus of an ankyrin repeat-containing siRNA binding protein in $w \mathrm{Ri}$. As is the case for 
178 many Wolbachia genomes, repetitive elements such as transposases and reverse transcriptases are

179 abundant in $w$ Rec and have hampered closing of the genome. 77 such repetitive genes were

180 found in $w$ Rec, and often appeared at the boundaries of scaffolds (Fig. 2). Although genomic

181 rearrangement between the genomes cannot be completely assessed because the $w$ Rec genome is

182 not closed, genes in $w$ Rec scaffolds were universally syntenic compared to $w$ Mel (Fig. 3), with

183 the exception of a 5kb region containing WD_0042-WD_0051 (WREC_0853-WREC_0863),

184 consisting of repetitive transposases, reverse transcriptases, hypothetical proteins, and 185 pseudogenes. This region would have been located on the first $w$ Rec scaffold if syntenic, but 186 instead is on scaffold 31 (Fig. 3).

\section{Prophage WO relics in the genome}

188 Whole-genome alignment of $w \mathrm{Rec}$ and $w \mathrm{Mel}$ revealed three major regions of genome 189 reduction, with $w$ Rec lacking a large portion of both phage WO regions present in $w$ Mel as well 190 as the entirety of the "Octomom" region (Chrostek et al., 2013) (Fig. 2), with only a bordering

191 reverse transcriptase, WREC_0508 (wMel homolog WD_0506) present. Interestingly, although

192 the minor capsid gene used in prior PCR surveys is absent, $w$ Rec does contain two major phage193 related regions (Fig. 2). The first is a 19.2kb region (WREC_0261-WREC_0285) across three

194 scaffolds that is homologous to 21 contiguous genes of wMel WO-A (WD_0276-WD_0296).

195 This region in $w$ Rec is syntenic and $99.4 \%$ identical to its homologous region in $w \mathrm{Mel}$, with two 196 exceptions. The $w$ Rec homolog (WREC_0270/WREC_0274) of WD_0285, an ankyrin repeat

197 protein, is fragmented by the insertion of two reverse transcriptases and a gap in the scaffolds,

198 and there is an 189bp insertion in WREC_0283 (WD_0294), another ankyrin repeat protein. If

199 these two regions are included in the calculation of similarity, then the $w$ Rec WO-A phage region 200 is $90.9 \%$ identical to the same region in $w \mathrm{Mel}$. The second $w \mathrm{Rec}$ phage region contains $11.3 \mathrm{~kb}$ 201 and 7 genes (WREC_0559-WREC_0568) that are syntenic and homologous to part of WO-B in 
202 w Mel (WD_0625-WD_0632), with 99.5\% pairwise identity. Two of these genes are interrupted

203 by premature stop codons and the remaining fragments are annotated as smaller, separate genes.

204 These genes include the orthologs of WD_0630, a hypothetical protein, which is split into three

205 genes in $w$ Rec (WREC_0563-WREC_0565), and the ortholog of WD_0632, which is split into

206 the 3,096bp gene WREC_0567 and 468bp gene WREC_0568. While the head region of WO

207 appears to be absent in $w$ Rec, the host adsorption module which is putatively involved in binding

208 to the host surface during phage infection is largely intact, as are a number of ankyrin repeat 209 genes, a transcriptional regulator, and the homologs of WP_02082/WP_0283, two genes in wPip

210 recently proposed as candidate mediators of CI (Beckmann and Fallon, 2013). In summary, the

211 markedly reduced number of phage genes in $w \operatorname{Rec}(\mathrm{N}=33)$ is the signature feature of the genome

212 compared to its closest relatives, which possess anywhere from 134 ( $w \mathrm{Ha})$ to 225 ( $w \mathrm{Ri}$ ) phage or 213 phage-associated genes (Fig. 4).

\section{Discussion:}

215 Divergence and genome reduction in wRec

216 Genome analysis revealed that $w \mathrm{Mel}$ and $w \mathrm{Rec}$ are very closely related with an average of

$21799.7 \%$ nucleotide identity in coding regions shared by the two strains. We estimate that $w$ Rec and

$218 w$ Mel diverged around 350,000 years ago. This estimate raises an interesting biogeographical

219 question: how could Wolbachia have been transferred at this time between the widely allopatric

220 Nearctic D. recens and Afrotropical D. melanogaster? Perhaps a widespread Drosophila-

221 generalist parasitoid played a role in vectoring this endosymbiont between host species, as

222 parasitoid wasps have been previously demonstrated as vectors for Wolbachia transfer (Heath et

223 al., 1999, Vavre et al., 2009). Molecular evidence suggests that the most recent Wolbachia sweep

224 within $D$. recens occurred only 50,000 years ago, while $D$. subquinaria split from $D$. recens an

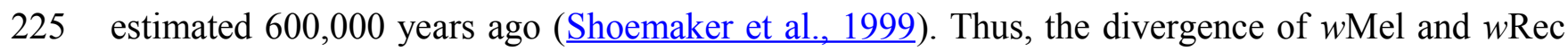


226 from their last common ancestor likely predated the most recent genetic sweep of $D$. recens, and

$227 w$ Rec infected $D$. recens after its incipient divergence from $D$. subquinaria (Werren and Jaenike,

228 1995, Shoemaker et al., 1999). Remarkably, these results suggest that $w$ Rec may have contributed

229 to reproductive isolation between these two species prior to the last glacial period $110,000-$

23012,000 years ago, when their ranges are thought to have been allopatric (Jaenike et al., 2006).

231 However, we note caution in interpreting the estimated divergence times as variability in

232 mutation rates between bacterial lineages can skew the estimates.

233 The four $w$ Rec genes evolving under positive selection are of particular interest as they

234 may be potential mediators of Wolbachia-host interactions (Table 2). Indeed, wsp is known to be

235 involved in pathogenicity and host interaction (Uday and Puttaraju, 2012) while OTU-like

236 cysteine proteases have deubiquitinase activity facilitating the pathogenicity of intracellular

237 pathogens and viruses (Furtado et al., 2013, Makarova et al., 2000). Although the function of the

238 hypothetical proteins is unknown, the presence of transmembrane (TM) domains suggests

239 interaction with the bacterial membrane and potentially its Drosophila host. Additionally, it has

240 previously been speculated that the elevated rate of mitochondrial DNA evolution in $D$. recens

241 was due to hitchhiking in association with a series of positive selection events in its resident

242 Wolbachia (Shoemaker et al., 2004).

243 The major difference between the $w \mathrm{Mel}$ and $w \mathrm{Rec}$ genomes was the incipient genome

244 reduction of WO prophage regions. Remaining phage WO genes in $w$ Rec were often bordered or

245 interrupted by transposases, suggesting that transposase activity may have been involved in the

246 removal and degradation of major portions of WO genomes. Over $100 \mathrm{~kb}$ of genetic material,

247 consisting mostly of phage-related genes, has likely been lost in $w$ Rec. Unlike the prophages

248 found in $w \mathrm{Mel}$ (Wu et al., 2004), all of $w$ Rec's WO regions lacked the head genes thought to be

249 necessary for mature virion formation (Metcalf and Bordenstein, 2012), including the orf7 minor

250 capsid protein used in previous PCR tests for WO (Bordenstein and Wernegreen, 2004). The lack 
251 of such head genes suggests that $w$ Rec has lost the capacity to serve as a source of WO phage to

252 infect other strains of Wolbachia. Future PCR screens may benefit from inclusion of more than 253 one primer set, perhaps adding primers for a gene from the host adsorption module, which is

254 highly conserved across WO prophages. However, it must be cautioned that the presence of 255 multiple and variable degenerate WO haplotypes makes it impossible for any set of one or two 256 primer pairs to detect all haplotypes.

Meanwhile, only 2,009 SNPs were present between the $w$ Mel and $w$ Rec genomes, 258 indicating that gene deletion has been heavily favored over mutation. Such genome reduction is 259 common in obligate intracellular bacteria, where many genes are expendable due to relaxed 260 selection and there is limited contact with novel gene pools (Casadevall, 2008). Given the 261 predatory nature of intact WO phages (Metcalf and Bordenstein, 2012, Bordenstein et al., 2006,

262 Sanogo and Dobson, 2006), it may have been evolutionarily advantageous for $w$ Rec to eliminate 263 the genes required for active phage production. It has been noted from TEM observations and 264 quantitative studies that WO phage can lyse Wolbachia, resulting in an inverse correlation 265 between bacterial and phage densities. Furthermore, because reproductive manipulations are 266 dependent on a critical density of Wolbachia, high phage activity correlates with low expression 267 of CI (Bordenstein et al., 2006). Since wRec exhibits high levels of CI in D. recens (Werren and 268 Jaenike, 1995), while $w \mathrm{Mel}$ shows lower levels of CI in D. melanogaster (Yamada et al., 2007), it 269 is possible that $w \operatorname{Rec}$ experiences a higher selective pressure to suppress phage, preserve high 270 bacterial densities, and maintain compatibility with its host's mating population. Thus, this 271 interaction could be one possible explanation for the major loss of phage genes in $w$ Rec that are 272 preserved in $w$ Mel.

Although Wolbachia has many more repetitive and mobile elements than most obligate 274 intracellular bacteria (Bordenstein and Reznikoff, 2005) and frequently switches hosts on an 275 evolutionary timescale (Vavre et al., 1999), it is worthwhile to note that there were only two 
genes in $w \operatorname{Rec}$ that were not present in $w \mathrm{Mel}$. It is possible that these genes were lost in $w \mathrm{Mel}$

277 after divergence from its last common ancestor with $w$ Rec.

278

279

280

281

282

283

284

285

286

287

288

289

290

291

292

293

294

295

296

297

298

299

The phage WO hypothesis to explain reproductive parasitism

Because the Octomom region was completely absent in $w$ Rec, it is unclear whether $w$ Rec lost these genes after diverging from $w \mathrm{Mel}$, or whether the genes were acquired by $w \mathrm{Mel}$ after divergence with their last common ancestor. Given that Octomom is not widespread in supergroup A Wolbachia, the latter possibility is likely. Moreover, although the function of Octomom in reproductive parasitism is unknown, it seems reasonable to conclude that the Octomom region is not needed for reproductive manipulations, as it is completely absent from $w$ Rec. Additionally, given the association of Octomom with increased Wolbachia virulence, proliferation, and host viral protection (Chrostek et al., 2013), we would predict that $w$ Rec would not possess these phenotypes, and may be a useful strain for confirming these associations.

It is intriguing that some WO genes are conserved in $w$ Rec while others were lost. One explanation for their preservation in $w$ Rec is that the remaining genes improve Wolbachia fitness.

Indeed, prophage sequences code for advantageous virulence factors in a wide array of bacterial species (Brussow et al., 2004). Because previous PCR surveys suggested $w$ Rec did not possess phage WO, speculation that WO may be involved in Wolbachia reproductive manipulations has been largely disregarded (Bordenstein and Wernegreen, 2004). However, our sequencing shows that although the phage genomes are not complete, $w$ Rec contains many phage-related genes including some that could be involved in CI and/or male-killing. These include at least four ankyrin repeat proteins, whose repetitive domain has been long thought to facilitate Wolbachiaeukaryote interaction (Iturbe-Ormaetxe et al., 2005, Siozios et al., 2013). Additionally, several WO genes in $w$ Rec are homologs of genes recently implicated in CI. WREC_0560 is a transcriptional regulator with $88.3 \%$ identity at the amino acid level to $w \operatorname{tr} M$ in $w$ PipMol, which 
increases expression of an important regulator of meiosis in Culex mosquitos and is postulated to

301 be a component of the molecular mechanisms of CI (Pinto et al., 2013). WREC_0566-

302 WREC_0568 meanwhile, are homologous to WP_0282 and WP_0283, two genes in $w$ Pip that

303 have been implicated in CI due to presence in the proteome of Wolbachia-infected, fertilized

304 mosquito spermathecae, along with their pattern of presence/absence in CI and non-CI strains

305 (Beckmann and Fallon, 2013). Although the $w$ Rec homolog of WP_0283 has been truncated by

$306427 \mathrm{bp}$, it has $99.8 \%$ nucleotide identity to the gene in $w \mathrm{Mel}$ (WD_0632) and an alternative

307 reading frame enables the transcription of the remaining nucleotides in the same frame as the C-

308 terminus of the homolog in $w$ Mel. Whether any of these WO genes are actually involved in

309 Wolbachia host manipulations remains unclear, especially since it is unknown whether the

310 remnants of phage WO are transcribed by $w$ Rec. However, the fact that these prophage regions

311 are conserved suggests that they may have a role to play in the biology of Wolbachia.

\section{WO host adsorption genes}

313 In addition to preservation of some potential reproductive manipulation mediators,

314 prophage WO genes WREC_0263-WREC_0269 contain an intact host adsorption module that

315 includes baseplate genes thought to be involved in the binding of WO to its bacterial host and

316 insertion of phage DNA. Indeed, this host adsorption module is nearly universal in WO prophage,

317 with very few degenerate phage haplotypes lacking these genes (Kent et al., 2011). Many

318 intracellular bacteria, including Wolbachia (Rances et al., 2008, Pichon et al., 2009), possess a

319 type IV secretion system that secretes effectors into the host as a common strategy to subvert

320 host-cell functions (Voth et al., 2012). A number of Gram-negative bacteria also possess a phage-

321 like type VI secretion system (Coulthurst, 2013); these include several obligatory intracellular

322 bacterial pathogens, such as Anaplasma and Ehrlichia (Rikihisa and Lin, 2010). Structural

323 analyses have shown this type of secretion system bears a remarkable resemblance to the spike 
324 protein of phages (Silverman et al., 2012). Given these similarities, and the fact that the WO host

325 adsorption module is almost universally present in sequenced arthropod Wolbachia (Kent et al..

326 2011), it is possible that Wolbachia may be using these genes to facilitate host-microbe

327 interactions, as a way to inject CI factors, genes, or other host manipulation particles into its host.

\section{Future Studies}

329 The discovery of phage elements in $w$ Rec opens up new questions. Additional

330 experiments will be needed to determine whether any conserved phage genes are involved in

331 Wolbachia manipulations of its host biology. In addition, we have seen that using single gene

332 markers of phage WO is not diagnostic of its absence. Thus, unsequenced Wolbachia strains that

333 were thought to be phage-free by PCR assays need reevaluation. Another question that remains is

334 how a single Wolbachia causes multipotent reproductive manipulations in different host

335 backgrounds. The availability of genomic sequence for a multipotent Wolbachia strain will enable

336 future transcriptomic and proteomic studies that could elucidate the genes involved in switching

337 reproductive phenotypes.

\section{Acknowledgements:}

339 We are grateful to Jennifer Wisecaver for technical assistance and to two reviewers for

340 their helpful feedback.

341 References:

342

343

344

345

346

347

348

349
ANGIUOLI, S. V., MATALKA, M., GUSSMAN, A., GALENS, K., VANGALA, M., RILEY, D. R., ARZE, C., WHITE, J. R., WHITE, O. \& FRICKE, W. F. 2011. CloVR: a virtual machine for automated and portable sequence analysis from the desktop using cloud computing. BMC Bioinformatics, 12, 356.

BALDO, L., DUNNING HOTOPP, J. C., JOLLEY, K. A., BORDENSTEIN, S. R., BIBER, S. A., CHOUDHURY, R. R., HAYASHI, C., MAIDEN, M. C., TETTELIN, H. \& WERREN, J. H. 2006. Multilocus sequence typing system for the endosymbiont Wolbachia pipientis. Appl Environ Microbiol, 72, 7098-110. 
364

365

366

367

368

369

370

371

372

373

374

375

376

377

378

379

380

381

382

383

384

385

386

387

388

389

390

391

392

393

394

395

396

397

398

399

400

401

BECKMANN, J. F. \& FALLON, A. M. 2013. Detection of the Wolbachia protein WPIP0282 in mosquito spermathecae: implications for cytoplasmic incompatibility. Insect Biochem Mol Biol, 43, 867-78.

BORDENSTEIN, S. R. \& BORDENSTEIN, S. R. 2011. Temperature affects the tripartite interactions between bacteriophage WO, Wolbachia, and cytoplasmic incompatibility. PLoS One, 6, e29106.

BORDENSTEIN, S. R., MARSHALL, M. L., FRY, A. J., KIM, U. \& WERNEGREEN, J. J. 2006. The tripartite associations between bacteriophage, Wolbachia, and arthropods. PLoS Pathog, 2, e43.

BORDENSTEIN, S. R. \& REZNIKOFF, W. S. 2005. Mobile DNA in obligate intracellular bacteria. Nat Rev Microbiol, 3, 688-99.

BORDENSTEIN, S. R. \& WERNEGREEN, J. J. 2004. Bacteriophage flux in endosymbionts (Wolbachia): infection frequency, lateral transfer, and recombination rates. Mol Biol Evol, 21, 1981-91.

BOYD, E. F. 2012. Bacteriophage-encoded bacterial virulence factors and phagepathogenicity island interactions. Adv Virus Res, 82, 91-118.

BRUSSOW, H., CANCHAYA, C. \& HARDT, W. D. 2004. Phages and the evolution of bacterial pathogens: from genomic rearrangements to lysogenic conversion. Microbiol Mol Biol Rev, 68, 560-602, table of contents.

BUSCHIAZZO, E., RITLAND, C., BOHLMANN, J. \& RITLAND, K. 2012. Slow but not low: genomic comparisons reveal slower evolutionary rate and higher $\mathrm{dN} / \mathrm{dS}$ in conifers compared to angiosperms. BMC Evol Biol, 12, 8.

CARVER, T., THOMSON, N., BLEASBY, A., BERRIMAN, M. \& PARKHILL, J. 2009. DNAPlotter: circular and linear interactive genome visualization. Bioinformatics, 25, 119-20.

CASADEVALL, A. 2008. Evolution of intracellular pathogens. Annu Rev Microbiol, 62, 19-33.

CHROSTEK, E., MARIALVA, M. S., ESTEVES, S. S., WEINERT, L. A., MARTINEZ, J., JIGGINS, F. M. \& TEIXEIRA, L. 2013. Wolbachia variants induce differential protection to viruses in Drosophila melanogaster: a phenotypic and phylogenomic analysis. PLoS Genet, 9, e1003896.

COULTHURST, S. J. 2013. The Type VI secretion system - a widespread and versatile cell targeting system. Res Microbiol, 164, 640-54.

DARBY, A. C., ARMSTRONG, S. D., BAH, G. S., KAUR, G., HUGHES, M. A., KAY, S. M., KOLDKJAER, P., RAINBOW, L., RADFORD, A. D., BLAXTER, M. L., TANYA, V. N., TREES, A. J., CORDAUX, R., WASTLING, J. M. \& MAKEPEACE, B. L. 2012. Analysis of gene expression from the Wolbachia genome of a filarial nematode supports both metabolic and defensive roles within the symbiosis. Genome Res, 22, 2467-77.

DARLING, A. E., MAU, B. \& PERNA, N. T. 2010. progressiveMauve: multiple genome alignment with gene gain, loss and rearrangement. PLoS One, 5, e11147.

DARRIBA, D., TABOADA, G. L., DOALLO, R. \& POSADA, D. 2012. jModelTest 2: more models, new heuristics and parallel computing. Nat Methods, 9, 772.

EDGAR, R. C. 2004. MUSCLE: multiple sequence alignment with high accuracy and high throughput. Nucleic Acids Res, 32, 1792-7.

FERRI, E., BAIN, O., BARBUTO, M., MARTIN, C., LO, N., UNI, S., LANDMANN, F., BACCEI, S. G., GUERRERO, R., DE SOUZA LIMA, S., BANDI, C., WANJI, S., DIAGNE, M. \& CASIRAGHI, M. 2011. New insights into the evolution of Wolbachia infections in filarial nematodes inferred from a large range of screened species. PLoS One, 6, e20843.

FOSTER, J., GANATRA, M., KAMAL, I., WARE, J., MAKAROVA, K., IVANOVA, N., BHATTACHARYYA, A., KAPATRAL, V., KUMAR, S., POSFAI, J., VINCZE, T., INGRAM, 
J., MORAN, L., LAPIDUS, A., OMELCHENKO, M., KYRPIDES, N., GHEDIN, E., WANG, S., GOLTSMAN, E., JOUKOV, V., OSTROVSKAYA, O., TSUKERMAN, K., MAZUR, M., COMB, D., KOONIN, E. \& SLATKO, B. 2005. The Wolbachia genome of Brugia malayi: endosymbiont evolution within a human pathogenic nematode. PLOS Biol, 3, el21.

FUJII, Y., KAGEYAMA, D., HOSHIZAKI, S., ISHIKAWA, H. \& SASAKI, T. 2001. Transfection of Wolbachia in Lepidoptera: the feminizer of the adzuki bean borer Ostrinia scapulalis causes male killing in the Mediterranean flour moth Ephestia kuehniella. Proc Biol Sci, 268, 855-9.

FURTADO, A. R., ESSID, M., PERRINET, S., BALANA, M. E., YODER, N., DEHOUX, P. \& SUBTIL, A. 2013. The chlamydial OTU domain-containing protein ChlaOTU is an early type III secretion effector targeting ubiquitin and NDP52. Cell Microbiol, 15, 2064-79.

GAVOTTE, L., HENRI, H., STOUTHAMER, R., CHARIF, D., CHARLAT, S., BOULETREAU, M. \& VAVRE, F. 2007. A Survey of the bacteriophage WO in the endosymbiotic bacteria Wolbachia. Mol Biol Evol, 24, 427-35.

GUEGUEN, G., ONEMOLA, B. \& GOVIND, S. 2012. Association of a new Wolbachia strain with, and its effects on, Leptopilina victoriae, a virulent wasp parasitic to Drosophila spp. Appl Environ Microbiol, 78, 5962-6.

HEATH, B. D., BUTCHER, R. D., WHITFIELD, W. G. \& HUBBARD, S. F. 1999. Horizontal transfer of Wolbachia between phylogenetically distant insect species by a naturally occurring mechanism. Curr Biol, 9, 313-6.

IOANNIDIS, P., DUNNING HOTOPP, J. C., SAPOUNTZIS, P., SIOZIOS, S., TSIAMIS, G., BORDENSTEIN, S. R., BALDO, L., WERREN, J. H. \& BOURTZIS, K. 2007. New criteria for selecting the origin of DNA replication in Wolbachia and closely related bacteria. BMC Genomics, 8, 182.

ITURBE-ORMAETXE, I., BURKE, G. R., RIEGLER, M. \& O'NEILL, S. L. 2005. Distribution, expression, and motif variability of ankyrin domain genes in Wolbachia pipientis. J Bacteriol, 187, 5136-45.

JAENIKE, J. 2007. Spontaneous emergence of a new Wolbachia phenotype. Evolution, $61,2244-52$.

JAENIKE, J., DYER, K. A., CORNISH, C. \& MINHAS, M. S. 2006. Asymmetrical reinforcement and Wolbachia infection in Drosophila. PLoS Biol, 4, e325.

KENT, B. N. \& BORDENSTEIN, S. R. 2010. Phage WO of Wolbachia: lambda of the endosymbiont world. Trends Microbiol, 18, 173-81.

KENT, B. N., FUNKHOUSER, L. J., SETIA, S. \& BORDENSTEIN, S. R. 2011. Evolutionary genomics of a temperate bacteriophage in an obligate intracellular bacteria (Wolbachia). PLoS One, 6, e24984.

KLASSON, L., WALKER, T., SEBAIHIA, M., SANDERS, M. J., QUAIL, M. A., LORD, A., SANDERS, S., EARL, J., O'NEILL, S. L., THOMSON, N., SINKINS, S. P. \& PARKHILL, J. 2008. Genome evolution of Wolbachia strain wPip from the Culex pipiens group. Molecular biology and evolution, 25, 1877-87.

KLASSON, L., WESTBERG, J., SAPOUNTZIS, P., NASLUND, K., LUTNAES, Y., DARBY, A. C., VENETI, Z., CHEN, L., BRAIG, H. R., GARRETT, R., BOURTZIS, K. \& ANDERSSON, S. G. 2009. The mosaic genome structure of the Wolbachia wRi strain infecting Drosophila simulans. Proceedings of the National Academy of Sciences of the United States of America, 106, 5725-30.

LIBRADO, P. \& ROZAS, J. 2009. DnaSP v5: a software for comprehensive analysis of DNA polymorphism data. Bioinformatics, 25, 1451-2.

MAKAROVA, K. S., ARAVIND, L. \& KOONIN, E. V. 2000. A novel superfamily of predicted cysteine proteases from eukaryotes, viruses and Chlamydia pneumoniae. Trends Biochem Sci, 25, 50-2. 
454

455

456

457

458

459

460

461

462

463

464

465

466

467

468

469

470

471

472

473

474

475

476

477

478

479

480

481

482

483

484

485

486

487

488

489

490

491

492

493

494

495

496

497

498

499

500

501

502

503

MASUI, S., KAMODA, S., SASAKI, T. \& ISHIKAWA, H. 2000. Distribution and evolution of bacteriophage WO in Wolbachia, the endosymbiont causing sexual alterations in arthropods. J Mol Evol, 51, 491-7.

METCALF, J. A. \& BORDENSTEIN, S. R. 2012. The complexity of virus systems: the case of endosymbionts. Curr Opin Microbiol, 15, 546-52.

MORENO-HAGELSIEB, G. \& LATIMER, K. 2008. Choosing BLAST options for better detection of orthologs as reciprocal best hits. Bioinformatics, 24, 319-24.

PICHON, S., BOUCHON, D., CORDAUX, R., CHEN, L., GARRETT, R. A. \& GREVE, P. 2009. Conservation of the Type IV secretion system throughout Wolbachia evolution. Biochem Biophys Res Commun, 385, 557-62.

PINTO, S. B., STAINTON, K., HARRIS, S., KAMBRIS, Z., SUTTON, E. R., BONSALL, M. B., PARKHILL, J. \& SINKINS, S. P. 2013. Transcriptional Regulation of Culex pipiens Mosquitoes by Wolbachia Influences Cytoplasmic Incompatibility. PLoS Pathog, 9, e1003647.

RANCES, E., VORONIN, D., TRAN-VAN, V. \& MAVINGUI, P. 2008. Genetic and functional characterization of the type IV secretion system in Wolbachia. J Bacteriol, 190, 5020-30.

RAYCHOUDHURY, R., BALDO, L., OLIVEIRA, D. C. \& WERREN, J. H. 2009. Modes of acquisition of Wolbachia: horizontal transfer, hybrid introgression, and codivergence in the Nasonia species complex. Evolution, 63, 165-83.

RIKIHISA, Y. \& LIN, M. 2010. Anaplasma phagocytophilum and Ehrlichia chaffeensis type IV secretion and Ank proteins. Curr Opin Microbiol, 13, 59-66.

RILEY, D. R., ANGIUOLI, S. V., CRABTREE, J., DUNNING HOTOPP, J. C. \& TETTELIN, H. 2012. Using Sybil for interactive comparative genomics of microbes on the web. Bioinformatics, 28, 160-6.

RONQUIST, F., TESLENKO, M., VAN DER MARK, P., AYRES, D. L., DARLING, A., HOHNA, S., LARGET, B., LIU, L., SUCHARD, M. A. \& HUELSENBECK, J. P. 2012. MrBayes 3.2: efficient Bayesian phylogenetic inference and model choice across a large model space. Syst Biol, 61, 539-42.

ROSS, C. L., DYER, K. A., EREZ, T., MILLER, S. J., JAENIKE, J. \& MARKOW, T. A. 2003. Rapid divergence of microsatellite abundance among species of Drosophila. Mol Biol Evol, 20, 1143-57.

SANOGO, Y. O. \& DOBSON, S. L. 2006. WO bacteriophage transcription in Wolbachiainfected Culex pipiens. Insect Biochem Mol Biol, 36, 80-5.

SANOGO, Y. O., EITAM, A. \& DOBSON, S. L. 2005. No evidence for bacteriophage WO orf7 correlation with Wolbachia-induced cytoplasmic incompatibility in the Culex pipiens complex (Culicidae: Diptera). J Med Entomol, 42, 789-94.

SARIDAKI, A., SAPOUNTZIS, P., HARRIS, H. L., BATISTA, P. D., BILISKE, J. A., PAVLIKAKI, H., OEHLER, S., SAVAKIS, C., BRAIG, H. R. \& BOURTZIS, K. 2011. Wolbachia prophage DNA adenine methyltransferase genes in different DrosophilaWolbachia associations. PLoS One, 6, e19708.

SHOEMAKER, D. D., DYER, K. A., AHRENS, M., MCABEE, K. \& JAENIKE, J. 2004. Decreased diversity but increased substitution rate in host mtDNA as a consequence of Wolbachia endosymbiont infection. Genetics, 168, 2049-58.

SHOEMAKER, D. D., KATJU, V. \& JAENIKE, J. 1999. Wolbachia and the evolution of reproductive isolation between Drosophilla recens and Drosophila subquinaria. Evolution, 53, 1157-1164.

SILVERMAN, J. M., BRUNET, Y. R., CASCALES, E. \& MOUGOUS, J. D. 2012. Structure and regulation of the type VI secretion system. Annu Rev Microbiol, 66, 45372.

PeerJ reviewing PDF | (v2014:07:2386:1:1:NEW 29 Jul 2014) 
504

505

506

507

508

509

510

511

512

513

514

515

516

517

518

519

520

521

522

523

524

525

526

527

528

529

530

531

532

533

534

535

536

537

538

539

540

541

542

543

544

545

SIOZIOS, S., IOANNIDIS, P., KLASSON, L., ANDERSSON, S. G., BRAIG, H. R. \& BOURTZIS, K. 2013. The diversity and evolution of Wolbachia ankyrin repeat domain genes. PLoS One, 8, e55390.

UDAY, J. \& PUTTARAJU, H. P. 2012. Comparative analysis of Wolbachia surface protein in D. melanoagster, A. tabida and B. malayi. Bioinformation, 8, 711-5.

VALLENET, D., ENGELEN, S., MORNICO, D., CRUVEILLER, S., FLEURY, L., LAJUS, A., ROUY, Z., ROCHE, D., SALVIGNOL, G., SCARPELLI, C. \& MEDIGUE, C. 2009. MicroScope: a platform for microbial genome annotation and comparative genomics. Database (Oxford), 2009, bap021.

VAVRE, F., FLEURY, F., LEPETIT, D., FOUILLET, P. \& BOULETREAU, M. 1999. Phylogenetic evidence for horizontal transmission of Wolbachia in hostparasitoid associations. Mol Biol Evol, 16, 1711-23.

VAVRE, F., MOUTON, L. \& PANNEBAKKER, B. A. 2009. Drosophila-parasitoid communities as model systems for host-Wolbachia interactions. Adv Parasitol, 70, 299-331.

VOTH, D. E., BROEDERDORF, L. J. \& GRAHAM, J. G. 2012. Bacterial Type IV secretion systems: versatile virulence machines. Future Microbiol, 7, 241-57.

WERREN, J. H., BALDO, L. \& CLARK, M. E. 2008. Wolbachia: master manipulators of invertebrate biology. Nat Rev Microbiol, 6, 741-51.

WERREN, J. H. \& JAENIKE, J. 1995. Wolbachia and cytoplasmic incompatibility in mycophagous Drosophila and their relatives. Heredity (Edinb), 75 ( Pt 3), 3206.

WERREN, J. H., ZHANG, W. \& GUO, L. R. 1995. Evolution and phylogeny of Wolbachia: reproductive parasites of arthropods. Proc Biol Sci, 261, 55-63.

WU, M., SUN, L. V., VAMATHEVAN, J., RIEGLER, M., DEBOY, R., BROWNLIE, J. C., MCGRAW, E. A., MARTIN, W., ESSER, C., AHMADINEJAD, N., WIEGAND, C., MADUPU, R., BEANAN, M. J., BRINKAC, L. M., DAUGHERTY, S. C., DURKIN, A. S., KOLONAY, J. F., NELSON, W. C., MOHAMOUD, Y., LEE, P., BERRY, K., YOUNG, M. B., UTTERBACK, T., WEIDMAN, J., NIERMAN, W. C., PAULSEN, I. T., NELSON, K. E., TETTELIN, H., O'NEILL, S. L. \& EISEN, J. A. 2004. Phylogenomics of the reproductive parasite Wolbachia pipientis wMel: a streamlined genome overrun by mobile genetic elements. PLoS Biol, 2, E69.

YAMADA, R., FLOATE, K. D., RIEGLER, M. \& O'NEILL, S. L. 2007. Male development time influences the strength of Wolbachia-induced cytoplasmic incompatibility expression in Drosophila melanogaster. Genetics, 177, 801-8.

YEN, J. H. \& BARR, A. R. 1971. New hypothesis of the cause of cytoplasmic incompatibility in Culex pipiens L. Nature, 232, 657-8.

ZHOU, W., ROUSSET, F. \& O'NEIL, S. 1998. Phylogeny and PCR-based classification of Wolbachia strains using wsp gene sequences. Proc Biol Sci, 265, 509-15.

ZUG, R. \& HAMMERSTEIN, P. 2012. Still a host of hosts for wolbachia: analysis of recent data suggests that $40 \%$ of terrestrial arthropod species are infected. PLoS One, 7, e38544. 


\section{Table 1 (on next page)}

$w R e c$ sequencing and genome statistics 


\begin{tabular}{|l|l|}
\hline Reads & $24,633,972$ \\
\hline Rec reads & $955,730(3.9 \%)$ \\
\hline Contigs & 159 \\
\hline Scaffolds & 43 \\
\hline Average coverage & 76.5 \\
\hline Genome size & $>1,126,653 \mathrm{bp}$ \\
\hline GC content & $35.2 \%$ \\
\hline CDS on scaffolds & 1271 \\
\hline Average CDS length & $764 \mathrm{bp}$ \\
\hline Average intergenic length & $130 \mathrm{bp}$ \\
\hline Transfer RNA's & 34 \\
\hline Ribosomal RNA's & $3(23 \mathrm{~S}, 16 \mathrm{~S}, 5 \mathrm{~S})$ \\
\hline Prophage regions & 2 \\
\hline
\end{tabular}




\title{
Table 2 (on next page)
}

$w$ Mel genes with less than $98 \%$ nucleotide identity to their orthologs in $w$ Rec

\begin{abstract}
Genes with a Ka/Ks ratio greater than one are highlighted.
\end{abstract}




\begin{tabular}{|c|c|c|c|c|c|c|c|c|c|}
\hline wMel Locus & $w$ Rec Locus & Function & $\begin{array}{c}\text { Pairwise } \\
\text { Identity } \\
(\%)\end{array}$ & $\begin{array}{l}w \text { Rec } \\
\text { length }\end{array}$ & $\begin{array}{l}\text { wMel } \\
\text { length }\end{array}$ & \# SNPs & $\begin{array}{c}\text { \# Non- } \\
\text { synonymou } \\
\text { s SNPs }\end{array}$ & $\begin{array}{l}\mathrm{Ka} / \mathrm{Ks} \\
\text { Ratio }\end{array}$ & $\begin{array}{c}\begin{array}{c}\text { Other } \\
\text { changes }\end{array} \\
\end{array}$ \\
\hline WD_0294 & 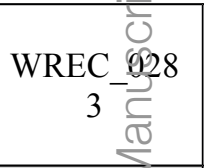 & $\begin{array}{l}\text { Ankyrin } \\
\text { Repeat } \\
\text { Domain } \\
\text { Protein }\end{array}$ & 89.4 & 1815 & 1626 & 4 & 4 & - & $\begin{array}{l}189 \mathrm{bp} \\
\text { insertion }\end{array}$ \\
\hline WD_0443 & WREC 244 & $\begin{array}{l}\text { OTU-like } \\
\text { Cysteine } \\
\text { Protease }\end{array}$ & 97.1 & 927 & 906 & 7 & 6 & 1.59 & $\begin{array}{l}21 \mathrm{bp} \\
\text { insertion }\end{array}$ \\
\hline WD_0550 & WREC_ & $\begin{array}{c}\text { Ankyrin } \\
\text { Repeat } \\
\text { Domain } \\
\text { Protein *TM } \\
\text { Domains } \\
\end{array}$ & 87.4 & 789 & 990 & 2 & 2 & - & $\begin{array}{c}99 \mathrm{bp} \\
\text { deletion, C- } \\
\text { terminal } \\
\text { frameshift, } \\
\text { alternate } \\
\text { start/stop } \\
\text { sites }\end{array}$ \\
\hline WD 0722 & $\begin{array}{c}\text { WREC_064 } \\
9\end{array}$ & $\begin{array}{c}\text { Hypothetical } \\
\text { Protein } \\
* \text { TM } \\
\text { Domains }\end{array}$ & 92.0 & 462 & 450 & 25 & 21 & 4.25 & $\begin{array}{c}9 \mathrm{bp} \\
\text { insertion, } 3 \\
\text { bp insertion }\end{array}$ \\
\hline WD_0996 & $\begin{array}{c}\text { WREC_095 } \\
6\end{array}$ & Transposase & 89.1 & 744 & 801 & 1 & 0 & 0 & $\begin{array}{l}\text { alternate } \\
\text { start site, } \\
\text { transposase } \\
\text { insertion }\end{array}$ \\
\hline WD_1007 & $\begin{array}{c}\text { WREC_097 } \\
3\end{array}$ & $\begin{array}{l}\text { Hypothetical } \\
\text { Protein }\end{array}$ & 95.1 & 366 & 351 & 3 & 2 & 0.42 & $\begin{array}{c}15 \mathrm{bp} \\
\text { insertion }\end{array}$ \\
\hline WD_1039 & $\begin{array}{c}\text { WREC_100 } \\
7\end{array}$ & $\begin{array}{c}\text { Collagen } \\
\text { Triple Helix } \\
\text { Repeat } \\
\text { Protein } \\
\end{array}$ & 97.5 & 405 & 1425 & 1 & 1 & - & $\begin{array}{c}9 \mathrm{bp} \\
\text { insertion, } \\
\text { scaffold } \\
\text { break }\end{array}$ \\
\hline WD_1063 & $\begin{array}{c}\text { WREC_103 } \\
6\end{array}$ & $\begin{array}{l}\text { Wsp Surface } \\
\text { Antigen }\end{array}$ & 97.9 & 708 & 714 & 9 & 8 & 2.55 & $\begin{array}{c}6 \mathrm{bp} \\
\text { deletion }\end{array}$ \\
\hline
\end{tabular}




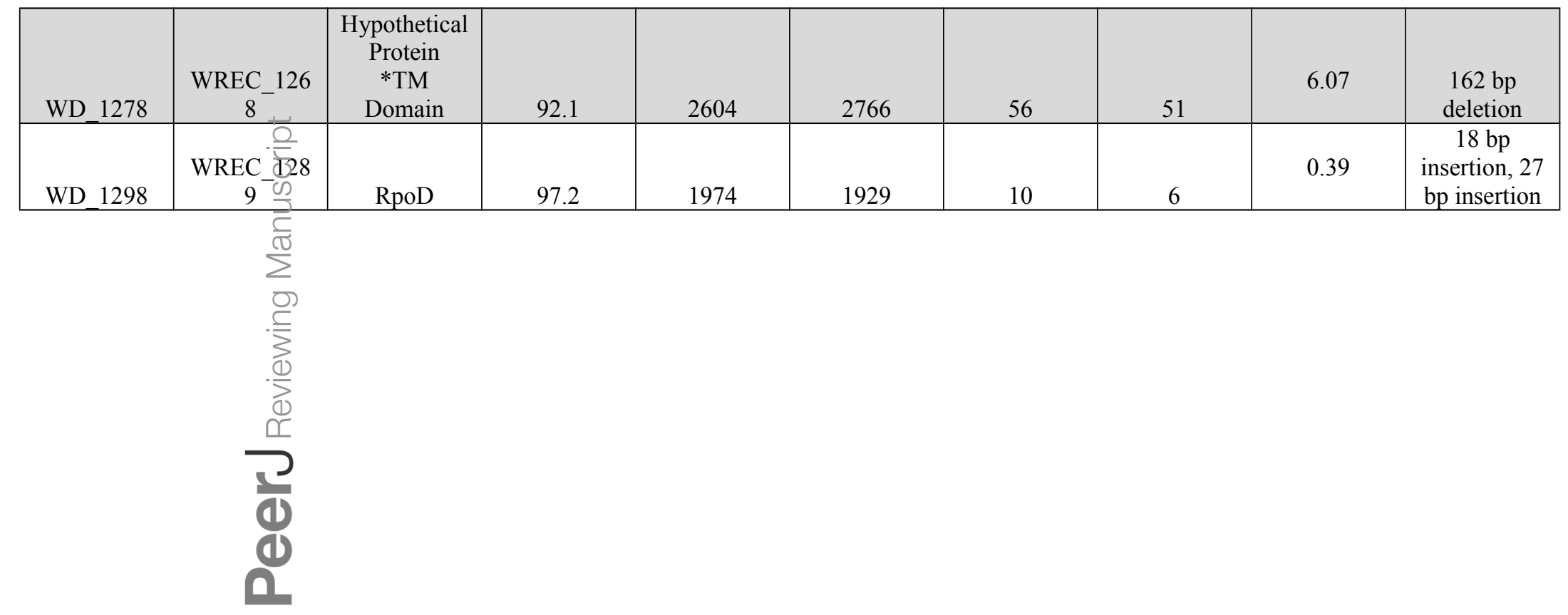




\title{
Figure 1
}

WO phage is present in all sequenced supergroup A and B Wolbachia strains

\begin{abstract}
A Bayesian phylogeny based on the concatenated Wolbachia multi-locus sequence typing genes is shown, consisting of selected strains with partial or full genome sequences and $w R e c$. All branches had posterior probabilities of $99 \%$ or greater. While all previously sequenced Wolbachia strains in group A and B possess phage WO elements, wRec (indicated with arrow), was formerly thought to be phage-free.
\end{abstract}




\section{Phage WO present}
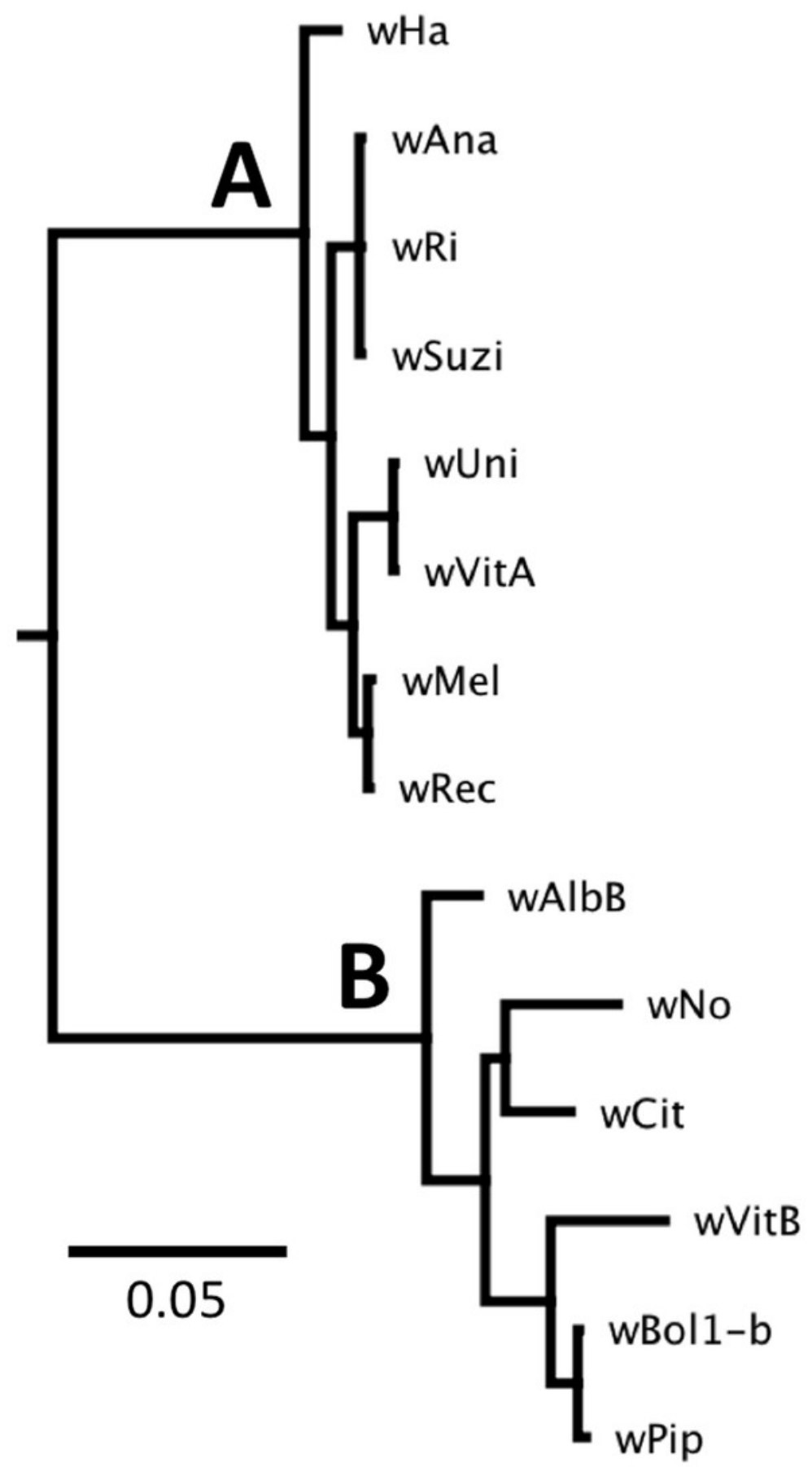


\section{Figure 2}

$w$ Rec genome comparison to $w \mathrm{Mel}$

$w$ Rec scaffolds were concatenated in the order in which their genes appear in wMel to produce the circular genome above. Major regions of loss or gain compared to wMel are indicated outside the circle along with the number of genes involved. $w \operatorname{Rec}$ genome features are indicated within the circle plot as follows (from outside-in): 1 (black): CDS in forward direction, and (magenta) genes not found in wMel; 2 (grey): CDS in reverse direction; 3 (red): scaffold break points; 4 (blue): WO regions; 5 (green): transposases and reverse transcriptases; 6 (purple/gold): GC content variation from average. WO prophage and related regions are shown and genes are categorized by color according to their likely functions and presence/absence in $w$ Rec. Locus tags for selected genes are indicated and dashed lines indicates breaks between scaffolds containing WO-A. The minor capsid gene of WO-A, which was used for prior PCR screens, is indicated with an asterisk. 


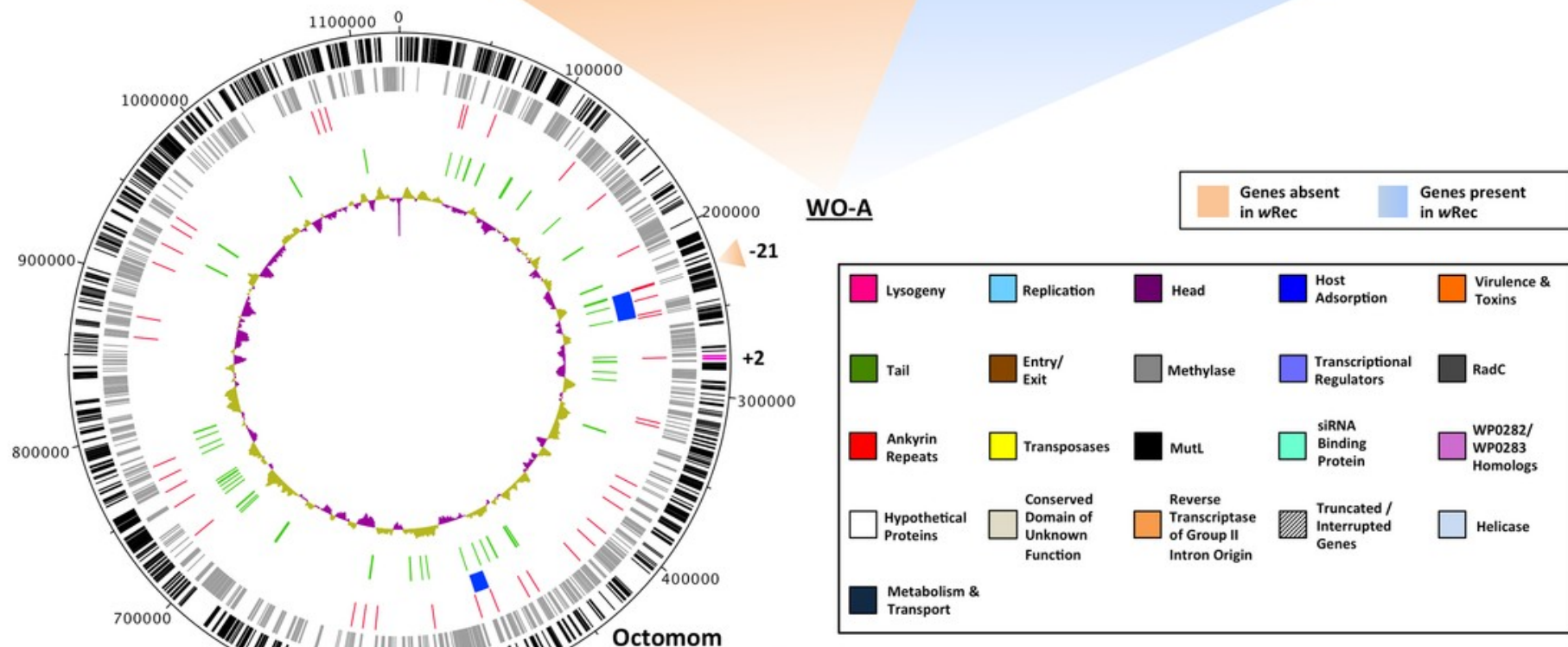

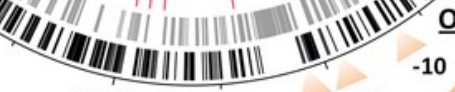

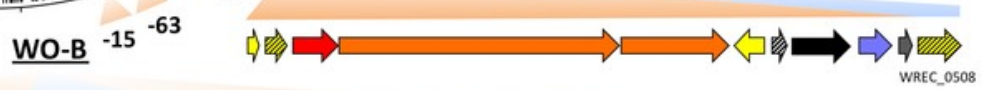

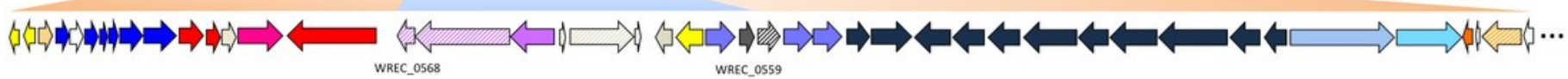
... 


\section{Figure 3}

Within-scaffold wRec synteny compared to wMel

$w$ Rec scaffolds were concatenated in the order in which they appear in wMel and withinscaffold synteny was analyzed. Genes are graphed as tick marks colored on a gradient from yellow to blue from left to right with wMel as the reference genome and each $w$ Rec gene colored according to the location of its homolog in the $w$ Mel genome. White spaces in $w$ Rec alone indicate the absence of homologous genes or genes with multiple paralogs whose synteny cannot be established, while white spaces shared by both genomes indicate intergenic regions. A $5 \mathrm{~kb}$ region of rearrangement consisting of repetitive elements and hypothetical proteins is noted with an arrow, and phage-related regions are marked.

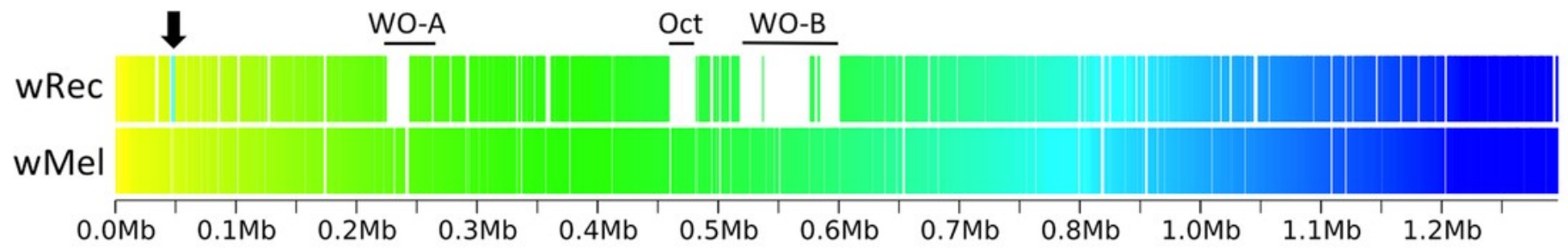




\section{Figure 4}

Number of phage genes in $w \operatorname{Rec}$ and its relatives

The total number of prophage, phage-associated, and WO-like island genes in each Wolbachia genome is plotted above a Bayesian phylogeny of their MLST genes. The approximate total length of phage genes in each genome is noted above each bar. 


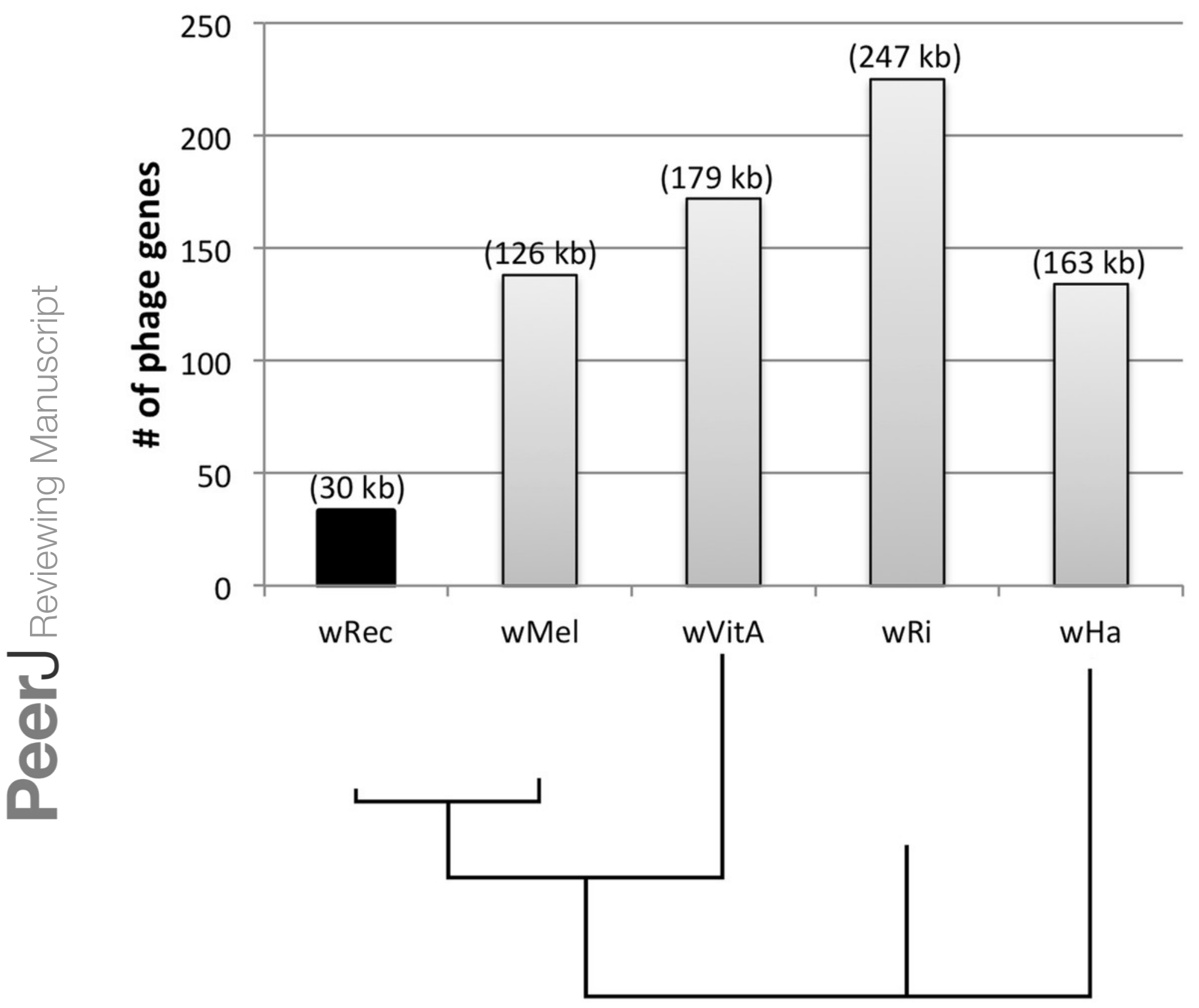

